\title{
Desenvolvimento da Compreensão Emocional
}

\author{
Maria da Glória Salazar d' Eça Costa Franco ${ }^{1}$ \\ Natalie Nobrega Santos \\ Universidade da Madeira
}

\begin{abstract}
RESUMO - A compreensão emocional é uma componente-chave da competência emocional, que se tem vindo a constatar ser importante para um melhor entendimento do desenvolvimento infantil. Este artigo faz uma revisão teórica desse construto, situando-o nos estudos sobre inteligência emocional e clarificando a sua definição. Apresentam-se, igualmente, os diferentes instrumentos que existem para a sua avaliação assim como os estudos que trazem evidências sobre o seu desenvolvimento. Concluiu-se que está demonstrada a existência de importantes mudanças na compreensão emocional da criança entre os 18 meses e os 12 anos. No entanto, é ainda necessário: a) reduzir a inconsistência conceptual presente nesse construto; b) determinar o que prediz; c) determinar se o seu ensino tem um efeito desejável no comportamento dos indivíduos.
\end{abstract}

Palavras-chave: compreensão emocional, inteligência emocional, desenvolvimento

\section{Emotional Comprehension Development}

\begin{abstract}
Emotional understanding is a key component of emotional competence, which has been singled out to be important for a better comprehension of child development. This paper is a theoretical review of this construct, placing it in the studies of emotional intelligence and clarifying its definition. It also presents the different existing tools towards its assessment as well as the array of studies providing evidence on its development. This theoretical research paper accounts for significantchanges inemotional understandingof childrenaged between 18 monthsand 12 years. Nonetheless, it is still necessary to: a) reduce theconceptualinconsistencyevidenced in thisconstruct; b) determine what it predicts; c) give an account whether its teachinghas adesirable impact on subjects' behaviour.
\end{abstract}

Keywords: emotional comprehension, emotional intelligence, development

A definição do construto de inteligência emocional (IE), como o resultado profícuo da interação entre as emoções e as cognições (Mayer \&Salovey, 1997), proporcionou uma nova forma de considerar o debate acerca da importância da emoção nas operações mentais (Mayer, Roberts, \& Barsade, 2008). Nessa perspectiva considera-se que: 1) a emoção torna o pensamento mais inteligente; 2) a inteligência cognitiva auxilia o indivíduo a pensar as suas emoções e as dos outros; 3) a ausência dessa relação binomial torna o indivíduo emocional e socialmente incapaz (Mayer \& Salovey, 1997). Essa valorização permitiu dirigir a atenção para a importância das emoções em vários contextos do desenvolvimento humano, tais como a família, a escola e o trabalho (Mayer et al., 2008).

Os três principais modelos de inteligência emocional - representados por Mayer, Salovey e Caruso; Bar-On; e Boyatzsis e Goleman - foram desenhados principalmente para o pensamento adulto, não para crianças. Todavia, crianças com alguns dias de vida são capazes de perceber emoções,e o desenvolvimento de habilidades emocionais continua durante toda a infância e vai até à idade adulta (Mayer \& Salovey, 1997).

Atualmente, os investigadores da psicologia clínica, social, educacional e do desenvolvimento têm começado a interessar-se pelo estudo da IE em crianças pequenas, propondo diferentes teorias explicativas (Scharfe, 2000). No

1 Endereço para correspondência: Maria da Glória Franco, Universidade da Madeira, Colégio dos Jesuítas - Rua dos Ferreiros, Funchal, Portugal. CEP: 9000-082.E-mail: gloria@uma.pt entanto, poucos investigadores abordaram especificamente o desenvolvimento da inteligência emocional, à exceção notável de Carolyn Saarni. Na revisão da literatura efetuada verificou-se a existência de muita inconsistência quer na terminologia, quer nos instrumentos de medida utilizados (Humphrey et al., 2011). Contudo, a maioria dos autores utiliza o termo competência emocional para referir-se à inteligência emocional no contexto do desenvolvimento infantil (Denham, Zinsser, \& Bailey, 2011).

Os investigadores concordam que a competência emocional é um construto desenvolvimental que abrange as habilidades das crianças para expressar adequadamente, interpretar e regular suas emoções, bem como para entender as emoções dos outros. No entanto, o número dos componentes que compõem a competência emocional varia de autor para autor. Por exemplo, Saarni identifica oito componentes (Schaffer, 2004), enquanto Mayer e Salovey (1997) identificam quatro. As habilidades mais consistentemente estudadas pelos autores no âmbito da literatura do desenvolvimento podem ser agrupadas em três componentes identificadas por Denham et al. (2003): A expressão emocional, a regulação emocional e a compreensão das emoções.

A expressão emocional assume um papel central na competência emocional. Essa componente refere-se à habilidade para moderar emoções negativas e potenciar emoções positivas, sem reprimir ou exagerar a informação que elas transmitem (Mayer \& Salovey, 1997). A expressividade positiva assume um papel fundamental na iniciação e na regulação das interações sociais, enquanto o afeto negativo 
pode comprometer a qualidade da interação com os pares (Denham et al., 2003).

A regulação das emoções refere-se à capacidade de modular a intensidade ou a duração dos estados emocionais. Todas as sociedades têm as suas regras para a regulação da emoções, como por exemplo, o que é aceitável na forma como são expressos os sentimentos emocionais. Isso aplica-se obviamente à agressão, que precisa de ser inibida e canalizada para que não seja prejudicial à vida social. No entanto, isso também se aplica às emoções positivas, como a alegria e o orgulho. Em algumas culturas manifestar a expressão de tais sentimentos também é desaprovado e desencorajado. As crianças devem, por isso, aprender a dissociar sentimentos internos de sua expressão aberta, e descobrir como fazê-lo é parte importante da sua socialização (Schaffer, 2004).

A compreensão das emoções refere-se à capacidade de identificar, reconhecer e nomear emoções; diferenciar as próprias emoções; compreender as emoções dos outros com base nas expressões faciais e nas características das situações de contexto emocional (Denhamet al., 2003). As crianças precisam aprender que sob certas circunstâncias elas ficam com raiva (ou medo, ou tristes, etc.), e quais são essas circunstâncias; como se sente cada emoção por dentro; como se expressa por fora, e que rotulo dar-lhe, a fim de falar sobre isso. Tudo isso envolve um grau de autoconsciência, isso é, a capacidade de se afastar e monitorar os próprios sentimentos e comportamentos - uma realização sofisticada na sua forma plenamente desenvolvida, embora o seu início já possa ser encontrado numa idade muito precoce (Schaffer, 2004).

Para Denham et al. (2003), a compreensão emocional é uma das componentes chave da competência emocional e existem evidências empíricas que a apontam como sendo preditora do ajustamento social e da saúde mental (Izard et al., 2001; Schultz, Izard, Ackerman, \& Youngstrom, 2001; Southam-Gerow \& Kendall, 2002). Por meio dessa habilidade, a criança pode ter uma interação mais adequada e ajustada aos contextos sociais, tendo a possibilidade de comunicar estados emocionais próprios e de saber o que acontece aos outros, requisitos chaves nos processos de regulação emocional e social (Delgado \& Contreras, 2008; Schaffer, 2004).

Outros estudos demonstram também que as crianças com maior capacidade de reconhecer e nomear emoções são mais aceites pelos pares e têm melhores resultados acadêmicos (Denham et al., 2003; Izard, 2001). Da mesma forma, baixos níveis de regulação e compreensão emocional estão significativamente associados com relatos de problemas comportamentais de pais e professores (Kidwell et al., 2010). As crianças que interpretam as mensagens emocionais dos outros com maior precisão pontuam melhor nas escalas de aprovação social. Efetivamente, crianças mais conhecedoras das emoções dos outros serão mais capazes de negociar as situações com os seus pares, obtendo, assim, melhores resultados e um menor número de conflitos (Miller et al., 2005). Assim, a compreensão emocional assume um papel essencial tanto na gestão das emoções como no desenvolvimento da conexão entre emoção, cognição e padrões emocionais (Izard et al., 2001).

O estudo da relação entre a compreensão emocional e o desenvolvimento infantil pode ser essencial para ajudar a criança no seu desenvolvimento saudável. No entanto, os conhecimentos sobre a compreensão emocional carecem de sistematização, devido a problemas quer conceptuais quer metodológicos, e ao pouco suporte para a intervenção prática. Sendo assim, o objetivo deste estudo é efetuar uma revisão teórica dos últimos 20 anos de investigação, salientando o que se sabe sobre as componentes essenciais da compreensão emocional, destacando os instrumentos utilizados para a avaliação desse constructo e apresentando alguns conceitos e formulações importantes acerca do desenvolvimento da compreensão emocional da criança desde o seu nascimento até os 12 anos de idade.

A revisão de literatura foi realizada nas bases de dados: a Biblioteca de Conhecimento Online (B-On); na $\mathrm{Web}$ of Science, na EBSCO; na Scientific Electronic Library Online (SciELO) e no Repositório Científico de Acesso Aberto de Portugal (RCAAP). Na pesquisa foram utilizadas como palavras-chave: "Emotional Competence", "Emotion comprehension", "Emotion Understanding" e "Emotion Knowledge"; além das palavras-chave em português "Compreensão emocional" e "Conhecimento emocional". Essa pesquisa foi refinada com os descritores: "Child Development" e "Emotional Development". Uma vez realizada uma revisão geral dos resultados dessas pesquisas, foram selecionados os artigos mais citados e dentro desses os que faziam uma clarificação dos conceitos estudados.

\section{Definição de Compreensão Emocional}

A definição da Compreensão Emocional não está de todo livre de inconsistência conceitual. Segundo Lamb (2009), um grande obstáculo que surge quando se realiza uma revisão teórica desse constructo é a existência de múltiplas definições na literatura atual. Em particular, a distinção entre a compreensão emocional e o conhecimento emocional é vaga e imprecisa. Aparentemente, alguns autores utilizam esses termos como sinônimos, enquanto outros investigadores utilizam a mesma terminologia para descrever constructos distintos, tornando difícil comparar, contrastar e integrar os trabalhos de diversos investigadores dentro do campo.

Considerando a definição original de conhecimento emocional apresentada por Susanne Denham, que mais comumente inclui o reconhecimento de emoções, a resposta à emoção dos outros e a compreensão das situações emocionais (Denham, citado por Lamb, 2009). Considerando que a compreensão da emoção refere-se ao conhecimento consciente sobre os processos emocionais ou crenças sobre como as emoções trabalham (Izard\& Harris, 1995, citados por Lamb, 2009). O caminho mais lógico para distingui-los é que o conhecimento emocional é a habilidade sociocognitiva de reconhecer as emoções, compreender as respostas às emoções dos outros e às situações emocionais, enquanto a compreensão emocional abarca esse conhecimento e uma série de habilidades e compreensões relacionadas com a emoção como: a compreensão da relação entre emoções e outros estados mentais; o conhecimento de estratégias de regulação das emoções; e a compreensão de respostas emocionais ambivalentes (Pons, Harris, \& de Rosnay, 2004). 
Apesar de interpretações um pouco diferentes, a imagem da compreensão emocional que emerge é a de um constructo complexo que engloba várias componentes, incluindo os classificados como conhecimento emocional. Ao reunir um corpo substancial de pesquisas, Pons et al. (2004) catalogam pelo menos nove componentes diferentes de compreensão emocional em crianças: (1) o reconhecimento das emoções, baseado nas expressões faciais; (2) compreensão das causas externas das emoções; (3) compreensão do desejo despertado; (4) compreensão das emoções baseadas em crenças; (5) compreensão da influência da lembrança em circunstâncias de avaliação de estados emocionais; (6) compreensão das possibilidades de controlar as experiências emocionais; (7) compreensão da possibilidade de esconder um estado emocional; (8) compreensão da existência de múltiplas ou até contraditórias (ambivalentes) respostas emocionais; (9) compreensão de expressões morais. Essas componentes desenvolvem-se com a idade e a experiência em situações sociais. São essas as componentes da compreensão emocional que serão consideradas nesta revisão.

\section{Instrumentos de avaliação da Compreensão Emocional}

Até há pouco tempo, havia muito poucos instrumentos que avaliassem a compreensão emocional das crianças (Franco, 2008). No entanto, a avaliação dessa componente é importante tanto para fins de pesquisa como clínicos. Do ponto de vista da pesquisa, uma medida que seja capaz de avaliar adequadamente diferenças individuais na compreensão emocional pode levar a uma melhor compreensão do papel dessas competências na adaptação da criança ao seu ambiente.

De facto, a compreensão emocional parece ser essencial, tanto do ponto de vista intrapessoal (por exemplo,componentes específicos da compreensão emocional têm sido associados a problemas emocionais e ao déficit de atenção e hiperatividade, Lamb, 2009) como interpessoal (por exemplo, o reconhecimento das emoções é uma das poucas componentes da compreensão emocional que tem demonstrado a capacidade de predizer adequação social e comportamental, Izard et al., 2001; Schultz et al., 2001). De uma perspectiva clínica, a triagem de crianças com déficits na compreensão emocional pode ajudar os profissionais a identificar as crianças que são vulneráveis a distúrbios físicos e psicológicos, crianças que poderiam beneficiar de uma intervenção individualizada atempada (Lahaye, Luminet, Broeck, Bodart,\&Mikolajczack, 2010).

Apesar da Compreensão Emocional ser importante em todo o ciclo de vida, poucos são os instrumentos desenvolvidos para medir essa componente.Em uma metaanálise realizada por Humphrey et al. (2011), os autores apresentam os resultados de uma revisão sistemática de instrumentos de avaliação de habilidades sociais e emocionais para crianças e jovens.A revisão sistemática relatada nesse artigo identificou12 medidas com diferentes características de implementação e propriedades psicométricas. Os autores sentem que essa variabilidade, particularmente em relação às propriedades psicométricas, reflete o estado emergente do campo. Embora tenha havido um interesse na aprendizagem social e emocional ao longo das últimas duas décadas, as medidas de desenvolvimento têm lutado para manter o ritmo. Este facto é, sem dúvida, refletido pelo facto de que muitos relatórios de avaliações de programas de aprendizagem sociais e emocionais não apresentam qualquer resultado da avaliação das habilidades sociais e emocionais, utilizando, pelo contrário, indicadores de sucesso relacionados com IE, como reduções nos problemas de saúde mental.

Os instrumentos mencionados por Humphrey et al. (2011) que medem algumas componentes da compreensão emocional são: o Assessment of Children's Emotion Skills (ACES) de Schultz, Izard e Bear (2004) que mede tendências de atribuição emocional (raiva, felicidade e tristeza), em crianças entre os 4 e 8 anos; o Emotion Regulation Checklist (ERC) de Shields e Cicchetti (1997), que mede a negatividade e a regulação emocional em crianças entre os 6 e 12 anos por meio da informação dos pais; e o Diagnostic Analysis of Nonverbal Accuracy (DANVA) de Nowicki e Duke (1989), que permite medir em crianças desde os 4 anos o reconhecimento de faces de crianças e adultos e da linguagem não-verbal.

Em um estudo sobre a capacidade preditiva e a utilidade das habilidades emocionais em crianças (com idades entre os cinco e nove anos), Izard et al.(2001) utilizaram tarefas de reconhecimento de emoções e nomeação de emoções. O Izard's Emotional Knowledge Test (EKT; Izard et al., 2001). O resultado dessa investigação, prevê uma medida integrativa da IE em crianças pequenas (desde os 3-4 anos) baseada, particularmente, na percepção e na compreensão das emoções.

No Brasil, Siqueira, Barbosa, e Alves (1999) desenvolveram a Medida de Inteligência Emocional, uma escala de autorrelato para adolescentes composta por 59 itens, cuja solução fatorial resultou em cinco fatores interpretados segundo o modelo proposto por Goleman em 1995. Um desses fatores é o da autoconsciência, que inclui a avaliação de componentes da compreensão emocional como a facilidade de lidar com os próprios sentimentos no que se refere à identificação, nomeação, avaliação, reconhecimento e atenção a esses sentimentos (Siqueira et al., 1999).

Bueno (2008) também construiu o Teste de Inteligência Emocional para Crianças(TIEC) constituído por 58 itens, elaborado para a avaliação das quatro habilidades relacionadas com a inteligência emocional, segundo o modelo de Mayer e Salovey (1997).A avaliação é baseada no desempenho dos sujeitos em tarefas, cujos estímulos são apresentados em forma de histórias e os problemas em forma de perguntas sobre os acontecimentos narrados. O teste mede dois componentes gerais da inteligência emocional infantil: o experiencial e o estratégico. O primeiro abrange itens relativos à percepção de emoções e à utilização da emoção para facilitação do pensamento (que inclui a capacidade de associar emoções a sensações térmicas, gustativas, etc., e a eventos do quotidiano). A componente estratégica abrange itens relacionados com o conhecimento sobre diferentes emoções e a associação dessas com eventos desencadeadores, além do conhecimento de estratégias de regulação emocional (Pavarini, Loureiro, \& Souza, 2010). O instrumento apresenta bons indicadores de validade e fidedignidade e 
pode ser recomendado para uso em avaliações do fator geral relacionado com a inteligência emocional (Bueno, 2008).

Em Portugal, Machado et al. (2008) adaptaram o Affect Knowledge Test, desenvolvido por Denham em 1986. É um teste de conhecimento das emoções para crianças desde a idade pré-escolar. O material do instrumento incluiu fantoches com faces amovíveis representando cada uma das emoções básicas primárias (alegria, tristeza, raiva e medo). As tarefas incluídas nesse teste distribuem-se nas seguintes dimensões: 1) reconhecimento verbal e identificação nãoverbal da expressão emocional; 2) conhecimento das emoções desencadeadas em situações típicas e atípicas. Alves, Cruz, Duarte, e Martins (2008) traduziram a Escala de Avaliação do Conhecimento Emocional (EACE). Franco e Candeias (2008) também estão a desenvolver um teste de percepção emocional para crianças entre os 7 e 13 anos. Segundo os autores, este instrumento, apesar de apresentar uma estrutura que vai ao encontro ao esperado e de na sua globalidade ter um elevado índice de precisão, tem alguns problemas ao nível de alguns itens, que estão a ser melhorados por meio de mais estudos ligados à validade desse instrumento.

Os instrumentos anteriormente mencionados visam observar só algumas componentes particulares da compreensão emocional, como o reconhecimento das emoções, sendo poucos os instrumentos que permitem observar outras componentes como, por exemplo, a compreensão das expressões morais. Considerando essa lacuna, Pons et al. (2004) propuseram o Test of Emotion Comprehension (TEC). O TEC está dividido em blocos de histórias com uma ordem pré-estabelecida, apresentando os contextos que permitem medir as nove componentes da compreensão emocional. Para a cotação, é atribuído um ponto em cada um dos componentes respondidos corretamente. Cada criança poderá obter no máximo nove pontos e no mínimo zero.

As vantagens desse instrumento são várias: primeiro, o teste não se restringe a um ou dois aspetos da compreensão emocional. Em vez disso, oferece uma oportunidade para avaliar os nove aspetos mencionados anteriormente, todos eles relativamente bem documentados na literatura experimental. Segundo, é de fácil aplicação e muito atrativo para as crianças. Terceiro, é um dos poucos instrumentos desenhado para crianças pequenas, entre os 3 e os 11 anos. As propriedades psicométricas do TEC foram estudadas a partir das respostas de uma amostra de 100 crianças inglesas de três, cinco, nove e onze anos, equitativamente distribuídas por sexo em cada faixa etária, sendo priorizada a análise de estrutura fatorial e a consistência interna do instrumento (.67). Pons et al. (2004) também fizeram uma análise de escalonamento de Guttman, com um índice de reprodutibilidade de .90, que sugere que os nove componentes são dimensionáveis e que a escala é válida. Igualmente, foram conduzidas análises de teste-reteste aos 3 meses $\left(r_{(18)}=.84\right)$ e aos 13 meses $\left(r_{(40)}=\right.$ .68), sendo controlados os efeitos de género (Pons et al., 2004). Encontraram-se, também, correlações positivas entre o TEC e as habilidades de linguagem medidas pelo Test of Receptive Grammar, TORG $\left(r_{(80)}=.81\right.$ e $r_{(76)}=.52$ quando os efeitos da idade e género eram controlados, Farina eAlbanese, 2007) e também uma correlação positiva $\left.\left(r_{(16}\right)=0,63\right)$ entre $o$ TEC e o TOM Storybooks $\left(r s_{(58)}=.77, p<.01\right.$, controlando a idade $-r=.37, p<.01)$ (Molina et al., 2007). A versão italiana obteve bons níveis de precisão $(K R=.79)$ e a análise Mokken realizada indicou níveis aceitáveis de escalabilidade $(H=.40, r s=.79)$ (Albanese et al. 2007).

A versão computadorizada do TEC foi desenvolvida no Brasil a partir da versão original lápis/papel construída por Pons et al. (2004) (validação por Roazzi, Dias, Minervino, Roazzi, \& Pons, 2008). A partir dos itens já construídos para a versão papel/lápis, foi possível a operacionalização dos construtos para a construção dos itens computadorizados. $\mathrm{Na}$ versão informatizada, as figuras foram redesenhadas no software Corel Draw, mantendo o formato original e depois foram exportadas em formato jpg para acrescentar o movimento, além de acrescentar o som (histórias) e criar o software para a aplicação do teste. As histórias foram narradas por uma equipa do departamento de Art Mídia da Universidade Federal de Campina Grande. Assim, as histórias são apresentadas em formato digitalizado de voz natural masculina, sem sotaque (Minervino, Roazzi, Dias, \& Roazzi, 2010).

A aferição e validação da versão portuguesa do TEC estão a ser desenvolvidas a partir da versão brasileira. Santos (2012) encontrou níveis aceitáveis de precisão (KR $=.66)$ e Silva (2012)encontrou correlações com as escalas de adaptabilidade $(r=.22)$ e intrapessoal $(r s=.24)$ do questionário de inteligência emocional de Bar-On (EQ-i).

Apesar de apresentar várias vantagens, o TEC apresenta também limitações. A fim de manter o interesse das crianças e testá-las em todos os nove componentes, o teste é necessariamente breve. Do ponto de vista psicométrico, esse procedimento tem limitações importantes. No entanto, os resultados obtidos com este teste apresentam-se semelhantes aos relatados na literatura utilizando vários itens por componentes (Pons et al., 2004). Futuras investigações deverão comparar o comportamento das crianças nas diferentes medidas de cada componente.

\section{Desenvolvimento da Compreensão Emocional}

É evidente que a idade influi nos recursos de compreensão da emoção. De facto, ao crescer, as crianças tornam-se mais conscientes dos diferentes aspetos das suas experiências emocionais. O estudo de Pons et al. (2004) realizado com o TEC indicou que as crianças apresentam uma melhoria clara com a idade no desempenho dos nove componentes avaliados por este instrumento. Também o estudo de DeStasio, Fiorilli, Chicchio e Albanese (2007) analisou o papel desempenhado pela idade, a linguagem e a dimensão intelectual nãoverbal na previsão da compreensão emocional. Os sujeitos foram 103 crianças italianas de jardins-de-infância públicos com idades entre os 3 e os 5 anos. As crianças foram testadas individualmente, usando as medidas: Teste de Compreensão das emoções (TEC) (Pons et al., 2004), Matrizes Progressivas Coloridas(SPM) (Raven, 1984) e teste para a recepção da Gramática (Trog) (Bishop, 1989). Os principais resultados mostraram que, em cada modelo testado, a componente cognitiva não-verbal não tornou-se significativa depois de entrar a idade na equação. As crianças apresentam uma clara melhoria com a idade em cada componente da compreensão 
emocional, aumentando regularmente a pontuação geral com a idade. No entanto, como também foi observado por Ponsetal.(2004), as diferenças individuais são notáveis em qualquer idade. Estes achados sugerem que a compreensão emocional e os seus componentes estão principalmente relacionados com a habilidade verbal e a idade.

Noutra investigação de Albanese, De Stasio, Chiacchio, Riorilli e Pons (2010),366 crianças (182 meninas e 184 meninos)com idades entre os 3 e os 10 anos foram testados utilizando o Teste de Compreensão da Emoção e as Matrizes Progressivas Coloridas. Os dados obtidos suportam a investigação anterior, demonstrando o importante papel que a idade desempenha no processo de compreensão da emoção. Os autores descobriram também que fatores cognitivos não-verbais são preditores apenas nas fases mais complexas de compreensão emocional. Estes achados indicam que, em média, o reconhecimento de um padrão mais complexo emocional estaria em função não só de idade mas também do bom desenvolvimento cognitivo, em termos de capacidade de representação em encontrar os sinais certos para compreender a emoção (Albanese et al., 2010). Um aumento no pensamento analítico pode melhorar a capacidade de representação nos aspetos mais complexos de compreensão da emoção.

Estudos realizados em Portugal com o TEC também observaram uma influência da idade na compreensão das emoções, tanto em crianças do pré-escolar (Santos, 2012) como em crianças do ensino básico (Silva, 2012).

Assim, a idade tem demonstrado ter efeitos diretos e indiretos sobre a emoção pela mediação da cognição. Existem dados empíricos que permitem conhecer os principais marcos da trajetória do desenvolvimento da compreensão emocional. A seguir, é apresentado um resumo dos principais estudos que examinam as principais mudanças na compreensão emocional das crianças desde o nascimento até os 12 anos.

\section{Os Primeiros Três Anos de Vida}

Desde muito cedo, as crianças mostram uma atenção especial pelos rostos e pelas expressões faciais. Uma habilidade rudimentar de reconhecer as emoções dos outros aparece logo após o nascimento (Schultz, Izard, \& Abe, 2005). Algumas células no córtex temporal, uma área implicada no reconhecimento facial, estão desenvolvidas às seis semanas de idade e outras áreas do córtex temporal associada ao reconhecimento facial estão completamente maduras aos 6 meses de idade (Rodman, Skelly,\& Gross; Rodman, citados por Schultz et al., 2005). Além dessas capacidades inatas e rapidamente desenvolvidas, por meio da modelagem e exposição, a socialização do reconhecimento de emoções ocorre imediatamente após o nascimento. As crianças imitam expressões faciais e gestos desde os primeiros dias de vida (Field, Woodson, Greenberg, \& Cohen; Meltzoff \& Moore,citados por Schultz et al., 2005).

Segundo Hughes (2011), estudos entre culturas indicam que aos 18 meses as crianças já podem identificar emoções simples, inclusive quando os desejos dos outros são diferentes dos seus próprios. Os primeiros estudos a explorar o desenvolvimento emocional das crianças demonstraram que a compreensão das expressões faciais desenvolve-se em sequência: a compreensão da alegria e tristeza primeiro, seguidos da compreensão da raiva e surpresa (Izard, citado por Scharfe, 2000).

Mas essa capacidade aparentemente inata de perceber, comparar e imitar expressões faciais é rudimentar, em comparação com a capacidade de identificar com precisão o significado de expressões emocionais. A partir do primeiro ano de vida, as crianças são capazes de utilizar informação emocional para fazer escolhas. No entanto, elas estão limitadas a decidir, por exemplo, se abordar ou evitar certos estímulos (Scharfe, 2000). O desenvolvimento da linguagem no segundo e terceiro anos de vida muda a natureza do contexto de socialização emocional. Aos 3 anos de idade,aproximadamente $93 \%$ das crianças usam regularmente os rótulos de emoções primárias como alegre, triste, com raiva e medo (Ridgeway, Waters, \&Kuczaj, citados por Schultz et al., 2005).

Emoções dirigidas para o Self, como a vergonha e o orgulho, só se desenvolvem depois dos dois ou três anos. Essas emoções dependem da internalização dos padrões parentais de comportamento, e mesmo as crianças um pouco mais velhas, frequentemente não possuem a sofisticação cognitiva necessária para reconhecerem tais emoções e o que as provoca (Papalia, Olds, \& Feldman, 2001).

O reconhecimento das emoções proporciona a base para o desenvolvimento de outras componentes da compreensão emocional. Crianças que identificaram adequadamente as emoções em uma tarefa experimental aos 3 anos apresentaram uma melhor compreensão de experiências emocionais complexas, como a compreensão de emoções múltiplas aos 6 anos (Dunn, Brown, \& Maguire, 1995, citado por Schultz et al., 2005).

Entre os quatro e os seis anos, ocorre uma série de mudanças cognitivas e comportamentais relacionadas com a compreensão emocional (León \&Sierra, 2008). Nessa idade, as crianças também começam a frequentar o jardim-deinfância e o pré-escolar, eventos que levam a modificações nas formas de interação e socialização da criança.

Considerando o desenvolvimento da compreensão emocional como uma faceta do seu desenvolvimento cognitivo e social mais amplo,os vários aspetos do desenvolvimento da compreensão das emoções a partir dos três anos foram agrupados por Pons et al. (2004) em três fases hierarquicamente organizadas: fase externa, fase mental e fase reflexiva.

\section{Fase Externa (entre os 3 e os 6 anos)}

A primeira fase envolve a identificação de expressões emocionais, entendendo que fatores externos situacionais podem causar emoções, e percebendo que a lembrança de uma situação passada pode causar uma reação emocional (Tenenbaum, Visscher, Pons, \& Harris, 2004). Dentro dos processos cognitivos que se adquirem no período pré-escolar e que têm sido estreitamente associados ao desenvolvimento da compreensão das emoções, encontra-se o uso da teoria da mente, a representação e construção de teorias sobre a causa das emoções, a predição de estados emocionais, a imitação simbólica, o desenvolvimento da empatia, entre outros. Estes processos têm sido vinculados às funções executivas, as quais, por sua vez, dependem das capacidades de armazenamento e processamento da informação da memória 
de trabalho, pelo que o desenvolvimento da compreensão das emoções pode depender, em grande medida, das capacidades de processamento da informação permitidas pela função executiva e a memória de trabalho (León \&Sierra, 2008).

Segundo Denham (2007), a capacidade de nomear verbalmente as emoções e de as identificar, não verbalmente, aumenta dos dois aos quatro anos e meio de idade. Como já foi mencionado, entre os três e os quatro anos, na sua maioria, as crianças já conseguem reconhecer e nomear expressões emocionais, com base nas emoções básicas, quando lhes são apresentadas fotografias e, com três anos, reconhecem um mínimo de quatro, de entre cinco, emoções com base na sua expressão facial (Pons\& Harris, 2005). As expressões de alegria são discriminadas mais cedo e com maior facilidade pelas crianças, seguidas das de tristeza e, mais tarde, das expressões de raiva e surpresa. Crianças com cinco anos identificam as emoções (não verbalmente) com maior facilidade do que as nomeiam verbalmente. Contudo, nomeiam mais facilmente as emoções de alegria e de tristeza, comparativamente com as de raiva e medo, tendo maior dificuldade na nomeação das emoções de medo e nojo (Machado et al., 2008).

Segundo Machado et al. (2008), as crianças em idade préescolar também demonstram um conhecimento considerável acerca das causas e consequências das situações emocionais, centrando-se nas qualidades salientes das situações e, nas expressões emocionais dos indivíduos. Dunn (citado por Machado et al., 2008) observou um súbito aumento do interesse das crianças (três anos) pelas causas das emoções de outras pessoas, uma vez que nessas idades começam a compreender a conexão direta entre os elementos de uma situação e as emoções, antecipando, por exemplo, a emoção de alegria, quando se recebe algo de desejável e, a emoção de tristeza, quando se frustra esse desejo.

Compreendem, ainda, que as emoções podem ser consequência da interpretação de uma situação e não o mero produto mecânico dessa por si só. Tal requer uma teoria da mente que toma como referência não a realidade objetiva, mas sim as suas representações subjetivas, sabendo que são essas que guiam as emoções e os atos das pessoas. Porém, a maioria das crianças dessa idade, ainda, pensa que as suas ideias acerca do que é desejável e as suas crenças acerca do verdadeiro estado de coisas se aplicam a toda a gente. Crianças de quatro anos tendem a mencionar apenas fatores situacionais, negligenciando ou não explicitando a experiência mental da pessoa (e.g. está zangada, porque isso é só para as raparigas) (Machado et al., 2008).

Entre os 3 e os 6 anos, as crianças também começam a compreender a relação entre a memória e a emoção. Elas começam a compreender que a intensidade das emoções decresce com o tempo e que alguns elementos do presente podem relembrar e reativar emoções passadas (Pons et al., 2004). Em um estudo de Lagattuta, Wellman, e Flavell (1997), foram apresentadas quatro histórias a 48 crianças, entre os 4 e os 6 anos, nas quais o protagonista experienciava um acontecimento triste. Mais tarde, cada protagonista da história encontra um sinal visual que está relacionado com $\mathrm{o}$ anterior acontecimento triste. Foi dito às crianças que o protagonista estava triste e foi-lhes pedido para explicar a razão. Os resultados sugerem que as crianças apresentam um desenvolvimento substancial entre os 4 e os 6 anos na compreensão da influência da atividade mental nas emoções.

Portanto, essa primeira componente da compreensão emocional surge, nas populações ocidentais, entre os 4 e 5 anos, dependendo da compreensão das relações simples de causa e efeito (Tenenbaum et al., 2004).

\section{Fase Mental (entre os 5 e 9 anos)}

A segunda fase da compreensão emocional inclui a aprendizagem de emoções que resultam de crenças e desejos individuais, e da percepção de que existem diferenças entre as emoções reais e a parentes.

Por volta dos dois ou três anos, muitas crianças compreendem que as emoções são afetadas em consequência da obtenção daquilo que o individuo deseja ou não (eles são felizes se obtêm o que querem e tristes quando não) (Harris, 1989). Segundo Machado et al. (2008), as crianças só contemplam o facto de as outras pessoas terem desejos e crenças diferentes dos seus entre os quatros e os cinco anos, enquanto as crianças mais velhas, de seis anos, mostram um aumento progressivo de consideração pelo carácter subjetivo das emoções, o que se pode inferir pelas referências explícitas aos desejos e/ou crenças dos intervenientes das situações (e.g. está zangado porque não quer isso e quer outra coisa). Essa compreensão está fortemente relacionada com o desenvolvimento da compreensão dos estados mentais próprios e dos outros (Wellman, Cross, \& Watson, 2001).

No entanto, as crianças poderão não manifestar a sua capacidade de levar em conta a informação pessoal e os estados mentais de outras pessoas em situações de maior intensidade emocional. Quando aumenta a intensidade das emoções, as crianças tendem a focar-se em fatores internos associados aos seus próprios objetivos (Denham, citado por Machado et al., 2008). Muitas crianças de idade pré-escolar citam os mesmos temas e as mesmas pessoas como causas para a raiva e para a tristeza, compreendendo a ambivalência das situações e sabendo que ambas as emoções podem resultar de situações semelhantes: percebendo a obstrução dos seus objetivos pelo outro, ativa-se a raiva, enquanto a tristeza resulta de uma percepção mais solitária da incapacidade de atingir o objectivo (Fabes, Eisenberg, \& McCormick, citado por Machado et al., 2008).

Já aos 2 e 3 anos de idade, as crianças compreendem que as pessoas têm desejos, mas só aos 4 ou 5 anos começam a compreender que as pessoas têm crenças, e que essas crenças podem ser falsas (Wellman et al., 2001). Igualmente, entre os 4 e os 6 anos, as crianças começam a compreender que as crenças da pessoa, sejam falsas ou verdadeiras, podem determinara sua reação emocional a uma situação (Pons et al., 2004). Um estudo de Harris, Johnson, Hutton, Andrews e Cooke (1989) indica que as crianças de seis anos, mas não as de quatro, predizem as emoções em função do que a outra personagem acredita de uma situação. As crianças mais pequenas ignoram a crença falsa que medeia a emoção e atendem unicamente à congruência entre o desejo do individuo e a situação real.

$\mathrm{Na}$ adoção e compreensão das regras de exibição de emoções, as crianças passam por duas fases (Harris, 1989). $\mathrm{Na}$ primeira, em torno de três e quatro anos de idade, elas aprendem a disfarçar os seus verdadeiros sentimentos em 
determinadas circunstâncias, mas parecem fazer isso de uma forma semi automática, talvez sob a pressão dos adultos para parecerem educadas ou bem-comportadas. Nessas idades, elas mostram pouco apreço explícito à potencial discrepância entre a emoção real e a emoção expressa e elas não entendem que a exibição deliberada de emoção pode ser usada para enganar as outras pessoas, e não apenas para conformar as expectativas do adulto em relação a um comportamento aceitável.Aos seis anos de idade, uma compreensão do impacto enganoso de regras de exibição começa a aparecer. Este conhecimento não pode ser baseado apenas em uma análise de expressões faciais e de comportamento, mas depende um pouco da compreensão de estados mentais (Tenenbaum et al., 2004). Um estudo de Sidera, Amadó e Serrat (2011) teve como objetivo estudar a compreensão que as crianças têm de que as emoções fingidas não coincidem necessariamente com as emoções internas, e, portanto, que as emoções fingidas podem criar falsas crenças nos seus observadores. Os resultados mostraram que a maioria das crianças de 4 e 6 anos de idade eram capazes de distinguir entre as emoções fingidas e reais, e que as crianças mais velhas tiveram melhor desempenho do que as crianças mais novas. Sobre as crenças do observador, a maioria das crianças tinha dificuldade para aperceber-se sobre as possíveis consequências enganosas das suas emoções fingidas.

As habilidades conceituais necessárias para alcançar essa segunda fase da compreensão da emoção só se desenvolvem quando as crianças ocidentais estão entre cerca dos 6 e os 7 anos de idade (Tenenbaum et al., 2004).

\section{Fase Reflexiva (entre os 8 e 12 anos)}

A terceira fase, que inclui a compreensão das emoções múltiplas ou mistas, das emoções morais, como a culpa e o controlo mental da emoção, surge mais tarde cerca dos 8 e 9 anos em crianças de populações ocidentais (Tenenbaum et al., 2004).A compreensão das emoções mistas é difícil para as crianças pequenas porque elas devem ser capazes de entender como um evento pode ser avaliado de duas formas diferentes.

Segundo Harter e Whitesell (1989), as crianças adquirem entre os 4 anos e os 12 anos de idade a compreensão das emoções mistas, passando por cinco níveis de desenvolvimento. Em um primeiro nível as crianças menores de 5 anos não conseguem sequer reconhecer a sensação de duas emoções ao mesmo tempo, mesmo que sejam semelhantes (isso é, com a mesma qualidade, por exemplo, feliz e contente). Em um segundo nível aos sete anos, as crianças podem considerar que duas emoções podem estar presentes ao mesmo tempo, mas apenas se ambas forem positivas ou negativas e dirigidas ao mesmo alvo. Essas crianças ainda não compreendem emoções simultâneas face a duas situações diferentes ou sentir emoções contraditórias face a uma mesma situação. Em um terceiro nível, por volta dos 8 anos, as crianças começam a compreender que podem sentir dois sentimentos do mesmo tipo dirigidos a alvos diferentes. Pelos 10 anos, quarto nível, as crianças demonstram compreender a possibilidade de ter dois sentimentos opostos ao mesmo tempo, mas apenas se eles forem dirigidos para dois alvos diferentes. No último nível, quinto nível, por volta dos 11 ou 12 anos, as crianças são capazes de compreender e descrever sentimentos opostos face a um mesmo alvo.

Já Harris (1989) sugere que por volta dos 6 ou 7 anos, as crianças começam a desenvolver uma compreensão das emoções no contexto moral e a desenvolver juízos morais. Para entender emoções morais, não só as crianças têm de compreender as crenças e desejos de um personagem, como devem também compreender a ruminação da personagem sobre como ele ou ela vai ser julgada por outros (Tenenbaum et al., 2004). Aos 8 anos, a maioria das crianças compreende que uma pessoa pode sentir-se mal ao obter o que quer mas de forma desonesta, interiorizando assim emoções morais como vergonha, culpa ou orgulho.

Finalmente, a compreensão do controle da emoção é complexa porque envolve um entendimento de que pode redirecionar seus pensamentos. Segundo Harris (1989), para controlar uma emoção, não é suficiente pôr cara de contentamento. As crianças sugerem estratégias mais ativas. A mais popular entre as crianças mais pequenas é mudar a situação. Essa técnica parece indicar que elas reconhecem que um acontecimento posterior pode mitigar uma emoção negativa. Crianças mais velhas não abandonam essa técnica, no entanto, ela é refinada, as crianças começam a descrever como mitigar uma primeira emoção suprimindo os pensamentos que mantêm essa emoção.

Assim, para ter sucesso na terceira fase da compreensão emocional, as crianças têm necessidade de alargara sua compreensão de maneira a que a reflexão cognitiva e a avaliação influencie mas reações emocionais (Tenenbaum et al., 2004).

Em resumo, a revisão efetuada indica que a compreensão emocional segue um caminho claro de desenvolvimento. Os estudos de Pons et al. por meio do TEC (2004) descobriram três fases claramente identificáveis desse desenvolvimento, cada uma das quais implica o domínio de um conjunto de componentes. Além disso, as suas pesquisas indicaram que uma criança deve dominar uma fase antes que ela possa progredir para a próxima (Pons et al., 2004; Pons \& Harris, 2005). A primeira fase (que ocorre cerca dos 5 anos de idade) inclui a compreensão de três importantes aspetos externos da emoção: as suas causas situacionais, a sua expressão externa, e esses eventos ou objetos que servem como avisos e lembretes externos que reativam a emoção. A segunda fase (que ocorre perto dos 7 anos de idade) implica o domínio da natureza mental das emoções: a conexão com desejos e crenças, e a distinção entre a emoção expressa e sentida. Finalmente, a terceira fase (em torno dos 9 e 11 anos de idade) é caracterizada pela compreensão de como um indivíduo pode refletir sobre uma determinada situação a partir de perspectivas diferentes e, assim,desencadear simultaneamente sentimentos diferentes: sentimentos conflituantes, emoções morais, como culpa e vergonha, e a regulação cognitiva da emoção (Pons et al., 2004).

Vários estudos confirmam a validade desse modelo desenvolvimental em diferentes países (Albanese et al., 2007; Tenenbauum et al., 2004; Roazzi et al., 2008), mas em trabalhos mais recentes outros padrões parecem emergir. No estudo de Roazzi et al. (2008) - que apresentou os resultados preliminares do processo de validação para o português, comparando as similaridades e diferenças das respostas ao 
TEC dadas pelas crianças britânicas, italianas, quéchuas e brasileiras de escolas públicas e particulares - os resultados indicam que, de modo geral, há uma maior similaridade entre as respostas da amostra italiana com a brasileira, do que a brasileira com a britânica, enquanto as crianças quéchuas tiveram o menor percentual de identificação de emoções. Assim, percebe-se a consistência da compreensão das emoções nas diversas culturas, mas ocorre variação na sincronia da identificação e a idade, devido, provavelmente, a diferenças educacionais e culturais (Belachi \& Faria, 2010; Tenenbaum et al., 2004). Além disso, essas divergências e a alta variabilidade dos scores obtidos no TEC por crianças da mesma faixa etária (Pons et al., 2004; Pons \& Harris, 2005) também podem estar relacionadas a outras características pessoais.As diferenças individuais na compreensão emocional são observáveis desde muito cedo e tais diferenças individuais permanecem bastante marcadas ainda no final da infância. Há evidência crescente de que essas diferenças individuais estão ligadas às características das crianças e às diferenças individuais nas suas relações sociais com colegas e adultos (Pons \& Harris,2005).

\section{Conclusão}

Nessa revisão quisemos mostrar que muito já foi feito para compreender melhor como desenvolve-se a compreensão emocional, mas que o caminho a percorrer ainda é muito longo. Apesar de ainda haver inconsistências na definição da compreensão emocional, podemos concluir que a Compreensão Emocional pode ser definida como uma série de habilidades e mecanismos de processamento da informação emocional como: a compreensão da relação entre emoções e outros estados mentais; o conhecimento de estratégias de regulação das emoções e a compreensão de respostas emocionais ambivalentes (Pons et al., 2004). Também existem vários instrumentos que permitem avaliar alguns dos componentes da compreensão emocional, como o Izard's Emotional Knowledge Test e o Assessment of Children's Emotion Skills, mas só Test of Emotion Comprehension (TEC) está desenhado para crianças pequenas, entre os 3 e os 11 anos e não se restringe a um ou dois aspetos de CE. Em vez disso,oferece uma oportunidade para avaliar a CE das crianças nos nove aspetos mencionados anteriormente, todos eles relativamente bem documentados na literatura experimental, mas pouco estudados no seu conjunto.

Os resultados das investigações realizadas pela psicologia do desenvolvimento também indicam que: 1) as crianças mostram com a idade uma clara melhoria no desempenho das diferentes componentes da compreensão emocional; 2) podem ser identificadas três fases do desenvolvimento, sendo cada uma delas caracterizada pela emergência de três das nove componentes da compreensão emocional; e 3) essas fases são consistentes nas diversas culturas.

Alguns estudos também têm mostrado que a compreensão das situações emocionais e o reconhecimento das emoções são preditores da aceitação pelos pares e dos bons resultados académicos. No entanto, nessa área ainda deverá desenvolverse mais investigação, uma vez que as investigações têm incidido apenas sobre algumas componentes da compreensão emocional, ficando outras por estudar, como por exemplo, a compreensão da possibilidade de esconder um estado emocional ou das expressões morais. Também seria importante estudar a relação entre a compreensão emocional com outras componentes da competência emocional como a expressividade emocional e a regulação emocional. Assim como, estudar os processos subjacentes a essa compreensão emocional, como é adquirida e como pode ser desenvolvida; e determinar se o ensino da compreensão das emoções tem um efeito desejável no comportamento dos indivíduos e produz mudanças significativas no seu dia-a-dia

\section{Referências}

Albanese, O., De Stasio, S., Chicchio, C., Fiorilli, C., \& Pons, F. (2010). Emotion Comprehension: The Impact of Nonverbal Intelligence. The Journal of Genetic Psychology, 171(2), 101115.doi: 10.1080/00221320903548084

Albanese, O., Molina, P., Gavazzi, I. G., De Stasio, S., Farina, E., Fiorilli, C., Antoniotti, C., Arati, L., \& Di Chiacchio, C. (2007).The assessment of emotion understanding: the Italian standardization of the Test of Emotion Comprehension (TEC). In V. Zammuner, \& C.Galli (Eds.), Giornata di studio sulle emozioni (pp. 44-47). Padova: CLEUP.

Alves, D., Cruz, O., Duarte, C., \& Martins, R. (2008). Escala de Avaliação do conhecimento Emocional (EACE). In A.P. Noronha, C. Machado, L.S. Almeida, M. Gonçalves, S. Martins \& V. Ramalho (Eds.), Actas da XIII Conferência Internacional Avaliação Psicológica: Formas e Contextos (CD). Braga: Psiquilibrios.

Belacchi, C., \& Farina, E. (2010). Prosocial/Hostile Roles and EmotionComprehension in Preschoolers. Aggressive Behavior, 36, 371-389. doi: 10.1002/ab.20361

Bishop, D. V. M. (1989). Test for reception of grammar (2nd ed). Published by the author at Department of Psychology, University of Manchester, Manchester.

Bueno, J.M. (2008). Construção de um instrumento para avaliação da inteligência emocional em crianças. (Unpublished master's thesis). Universidade São Francisco, Itatiba.

De Stasio, S., Fiorilli, C., Di Chiacchio, C., \& Albanese, O. (2007). Children's emotion understanding: effects of age and language. In V. Zammuner, \& C. Galli (Eds.), Giornata di studio sulle emozioni (p. 90). Padova: CLEUP.

Delgado, B., \& Contreras, A. (2008). Desarrollo social y emocional. In B. Delgado (Ed.), Psicología delDesarrollo (vol. II, pp. 3566). Madrid: McGraw Hill.

Denham, S. (2007). Dealing with feelings: How children negotiate the worlds of emotions and social relationships. Cognitie, Creier, Comportament, 11(1), 1-48. Retrieved from http:// denhamlab.gmu.edu/Publications\%20PDFs/Denham\% 202007.pdf

Denham, S. A. (1986). Social cognition, social behavior, and emotion in preschoolers: Contextual validation. Child Development, 57, 194-201.

Denham, S., Blair, K., DeMulder, E., Levitas, J., Sawyer, K., Auerbach-Major, S., \&Queenan, P. (2003). Preschool emotional competence: pathway to social competence? Child development, 74(1), 238-256.doi: 0009-3920/2003/7401-0017 
Denham, S., Zinsser, K., \& Bailey, C. (2011). Emotional intelligence in the first five years of life.Encyclopedia on Early Childhood Development. Montreal: Centre of Excellence for Early Childhood Development and Strategic Knowledge.

Farina, E., \& Albanese, O. (2007). Children's emotion understanding: individual differences and pragmatic competences. In V. Zammuner \& C.Galli (Eds.), Giornata di studio sulle emozioni (pp. 105-108). Padova: CLEUP.

Franco, M. G. (2008). Inteligência emocional: O que se sabe hoje. In Candeias, A., Almeida, L., Roazzi, A \& Primi; R. (Eds.), Inteligência Múltiplos Enfoques. São Paulo: Casa do Psicólogo.

Franco, M., \& Candeias, A. (2008). Teste de Percepção Emocional para Crianças: Estudos exploratórios para uma população portuguesa. In A. P. Noronha, C. Machado, L. S. Almeida, M. Gonçalves, S. Martins, \& V. Ramalho (Eds.), Actas da XIII Conferência Internacional Avaliação Psicológica: Formas e Contextos (CD). Braga: Psiquilibrios.

Harris, P. (1989). Children and emotion. The development of psychological understanding. Oxford: Blackwell Publishers.

Harris, P.L., Johnson, C.N., Hutton, D., Andrews, G., \& Cooke, T. (1989). Young Children's Theory of Mind and Emotion (Abstract). Cognition \& Emotion, 3(4), 379-400.doi: 10.1080/02699938908412713

Harter, S., \&Whitesell, N. (1989). Developmental changes in children's understanding of single, multiple, and blended emotion concepts. In C. Saarni\& P. Harris (Eds.), Children's understanding of emotion (pp. 81-116). Cambridge: Cambridge University Press.

Hughes, C. (2011). Social Understanding and social lives. New York: Spychology press.

Humphrey, N., Kalambouka, A., Wigelsworth, M., Lendrum, A., Deighton, J., \&Wolpert, M. (2011) Measures of Social and Emotional Skills for Children and Young People: A Systematic Review. Educational and Psychological Measurement, 71(4), 617-637.doi: 10.1177/0013164410382896

Izard, C. (2001). Emotional intelligence or adaptive emotions? Emotion, 1(3), 249-257. doi: 10.1037//1528-3542.1.3.249

Izard, C., Fine, S., Schultz, D., Mostow, A., Ackerman, B., \&Youngstrom, E. (2001). Emotion Knowledge as a predictor of social behavior and academic competence in children at risk. Psychological Science, 12(1), 18-23.doi: 10.1111/14679280.00304

Kidwell, S., Young, M., Hinkle, L., Ratliff, D., Marcum, M., \& Martin, C. (2010). Emotional competence and behavior problems: Differences across Preschool Assessment of Attachment Classifications. Clinical Child Psychology and Psychiatry, 15(3), 391-406. doi: 10.1177/1359104510367589

Lagattuta, K. H., Wellman, H. M., \& Flavell, J. H. (1997). Preschoolers' Understanding of the Link between Thinking and Feeling: Cognitive Cuing and Emotional Change. Child Development, 68(6), 1081-1104.

Lahaye, M., Luminet, O., Broeck, N., Bodart, E., \& Mikolajczak, M. (2010). Psychometric Properties of the Emotion Awarenes Questionnaire for Children in a French-Speaking Population. Journal of Personality Assessment, 92(4), 317-326. doi: $10.1080 / 00223891.2010 .482003$
Lamb, B. (2009). My Friends always know when I'm sad: how children's emotion understanding is associated with socioemotional development (Unpublished master's thesis). Retrieved from Victoria University of Wellingtonhttp://hdl. handle.net/10063/1618

León, D., \& Sierra, H. (2008). Desarrollo de la comprensión de las consecuencias de las emociones. Revista latinoamericana de psicología, 40(1), 35-45. Retrieved from http://openjournal. konradlorenz.edu.co/index.php/rlpsi/article/viewFile/354/255

Machado, P., Veríssimo, M., Torres, N., Peceguina, I, Santos, A.J., \&Rolão, T. (2008). Relações entre o conhecimento das emoções, as competências académicas, as competências sociais e a aceitação entre pares. Análise Psicológica, 26(3), 463-478.

Mayer, J., \&Salovey, P. (1997). What Is Emotional Intelligence? In P. Salovey, \& D. Sluyter (Eds.), Emotional development and emotional intelligence.Educational Implications (pp. 3-31). New York: Basic Books.

Mayer, J., Roberts, R., \&Barsade, S. (2008). Human Abilities: Emotional Intelligence. Annual Review of Psychology, 59, 507-536.doi: 10.1146/annurev.psych. 59.103006.093646

Miller, A.L., Gouley, K.K., Seifer, R., Zakriski, A., Eguia, M., Vergani, M. (2005). Emotion Knowledge Skills in Low-income Elementary School Children: Associations with Social Status and Peer Experiences. Social Development, 14(4), 637-651. Doi: 10.1111/j.1467-9507.2005.00321.

Minervino, C.A., Roazzi, A., Dias, M.G., \& Roazzi, M. (2010). Versão computadorizada do Teste de Compreensão das Emoções. In M.C. Joly, \& C.T., Reppold (Ed.), Testes informatizados para a avaliação psicológica e educación (E-book). São Paulo: Casa do Psicólogo.

Molina, P., Bulgarelli, D., Arati, L., Gianotti, F., Cattich, F., Calizia, L., \&Blijd-Hoogweiss E. (2007). Developmental trend of children's responses to TEC and to ToM-Storybooks: a study on concurrent validity. In V.L. Zammuner, \& C.Galli (Eds.), Giornata di studio sulleemozioni (pp. 171-174).Padova: CLEUP.

Nowicki, S., Jr. \& Duke, M. P. (1989). A measure of nonverbal social processing ability in children between the ages of 6 and 10. Paper presented at the American Psychological Society. Alexandria,V.A.

Papalia, D., Olds, S., \& Feldman, R. (2001). O Mundo da Criança. Lisboa: McGraw-Hill.

Pavarini, G., Loureiro, C. P., \& Souza, D. H. (2010). Compreensão de Emoções, Aceitação Social e Avaliação de Atributos Comportamentais em Crianças Escolares. Psicologia: Reflexão e Crítica, 24(1), 135-143.

Pons, F., \& Harris, P. (2005).Longitudinal change and longitudinal stability of individual differences in children's emotion understanding. Cognition \& Emotion, 19(8),1158-1174. doi: 10.1080/02699930500282108.

Pons, F., Harris, P., \& de Rosnay, M. (2004). Emotion comprehension between 3 and 11 years: Developmental periods and hierarchical organization. European journal of developmental psychology, 1(2), 127-152.doi:10.1080/17405620344000022

Raven, J. C., Court, J. H. \& Raven, J. (1984). Manual for Raven's Progressive Matrices and Vocabulary Scales. Section 2: Coloured progressive matrices. London: H. K. Lewis. 
Roazzi, A., Dias, M., Minervino, C., Roazzi, M., \& Pons, F. (2008). Compreensão das emoções em crianças: estudo transcultural sobre a validação do teste de compreensão da emoção TEC (testofemotioncomprehension). In A. Noronha, C. Machado, L. Almeida, M. Gonçalves, S. Martins \& V. Ramalho (Eds.), Actas da XIII Conferência Internacional Avaliação Psicológica: Formas e Contextos (CD). Braga: Psiquilibrios.

Santos, N. N. (2012). Inteligência Emocional: A compreensão das emoções em crianças do pré-escolar (Unpublishedmaster's thesis). Universidade da Madeira, Funchal.

Schaffer, H. (2004). Introducing Child Psychology. Singapore: Blackwell Publishing.

Scharfe, E. (2000). Development of emotional expression, understanding, and regulation in infants and young children. In J. Parker \& D. Goleman (Ed.), The Handbook of Emotional Intelligence (pp. 244-262). San Francisco: Jossey-Bass.

Schultz, D., Izard, C., \& Abe, J. (2005). The emotion systems and the development of emotional intelligence. R. Schulze \& R. Roberts (Eds.), Emotional Intelligence (pp. 51-67). Massachusetts: Hogrefe \& Huber.

Schultz, D., Izard, C., Ackerman, B., \& Youngstrom, E. (2001). Emotion Knowledge in Economically Disadvantaged Children: Self-regulatory Antecedents and Relations to Social Difficulties and Withdrawal. Development and Psychopathology, 13(1), 53-67.doi: 10.1017/S0954579401001043
Shields, A. \& Cicchetti, D. (1997). Emotion regulation among school-age children: The development and validation of a new criterion Q-sort scale. Developmental Psychology, 33(6), 906-916. doi: 10.1037/0012-1649.33.6.906.

Sidera, F., Amadó, A., \& Serrat, E. (2011. September). Do young children understand that pretend emotions are not real? Paper presented at the meeting of III International Congress of Emotional Intelligence, Opatija. Retrieved from http://www. icei2011.org/pdf/ICEI2011-Book_of_Abstracts.pdf

Silva, E. P. (2012). A relação entre inteligência emocional e o rendimento escolar em crianças do 1 . $^{\circ}$ ciclo do ensino básico da R.A.M. (Unpublished master'sthesis). Universidade da Madeira, Funchal.

Siqueira, M. M., Barbosa, N. C., \& Alves, M. T. (1999). Construção e Validação Fatorial de uma Medida de Inteligência Emocional. Psicologia: Teoria e Pesquisa, 15(2), 143-152. Retrieved from http://www.scielo.br/pdf/ptp/v15n2/a07v15n2.pdf

Southam-Gerow, M. A., \& Kendall, P. C. (2002).Emotion Regulation and understanding implication for child psychopathology and therapy. Clinical Psychology Review, 22, 189-222.doi: 10.1016/ S0272-7358(01)00087-3

Tenenbaum, H., Visscher, P., Pons, F., \& Harris, P. (2004).Emotional Undestanding in Quechua Children from an agro-pastoralist village. International Journal of Behavioral Development, 28(5), 471-478.doi: 10.1080/01650250444000225

Wellman, H.M., Cross, D., \& Watson, J. (2001). Meta-analysis of theory-of-mind development: the truth about false belief. Child Development, 72(3), 655-684.

Recebido em 26.08.2013

Primeira decisão editorial em 03.12.2014

Versão final em 08.01.2015

Aceito em 12.03.2015 